\title{
Weight Distribution of Periodic Errors and Optimal/Anti-Optimal Linear Codes
}

\author{
Pankaj Kumar Das \\ Department of Mathematics, Shivaji College, University of Delhi, Raja Garden, New Delhi 110027, India \\ Correspondence should be addressed to Pankaj Kumar Das; pankaj4thapril@yahoo.co.in
}

Received 24 May 2014; Accepted 14 August 2014; Published 31 August 2014

Academic Editor: Shaofang Hong

Copyright (C) 2014 Pankaj Kumar Das. This is an open access article distributed under the Creative Commons Attribution License, which permits unrestricted use, distribution, and reproduction in any medium, provided the original work is properly cited.

\begin{abstract}
The paper discusses weight distribution of periodic errors and then the optimal case on bounds of parity check digits for $\left(n=n_{1}+n_{2}\right.$, $k$ ) linear codes over GF $(q)$ that corrects all periodic errors of order $r$ in the first block of length $n_{1}$ and all periodic errors of order $s$ in the second block of length $n_{2}$ and no others. Further, we extend the study to the case when the errors are in the form of periodic errors of order $r$ (and $s$ ) or more in the two subblocks.
\end{abstract}

\section{Introduction}

In coding theory, many types of error patterns have been considered, and codes accordingly are constructed to combat such error patterns. Periodic errors are one type of error patterns that are found in channels like astrophotography [1], gyroscope and computed tomography [2]. Such error occurs due to happening of disturbances periodically. So, there is a need to study such errors and to develop codes dealing with such errors. It was in this spirit that codes detecting/correcting such errors were studied by Das and Tyagi $[3,4]$. A periodic error of order $s$ is defined as follows.

Definition 1. A periodic error of order $s$ is a vector whose nonzero components are located at $s$ shifting positions in a code vector where $s=1,2,3, \ldots,(n-1)$ and the number of its starting positions is among the first $s+1$ components.

For $s=1$, the periodic errors of order 1 are the vectors where error may occur in 1st, 3rd, 5 th, ..., positions or 2 nd, 4 th, 6 th, ..., positions. For example, in a vector of length 8 , periodic errors of order 1 are of the type 10101000, 00101000, 0010101, 10101010, 10001010, 01010101，01000101，00000101, 00000001, and so forth.

For $s=2$, the periodic errors of order 2 may look like 10010010, 10000010, 00010010, 01001001, 01000001, 01000000, 00001001, and so forth in a vector of length 8 .
Perfect codes are the best codes among the linear codes since the parameters satisfy the Sphere-Packing (or Hamming) bound $[5,6]$. It was a big challenge for mathematician to search for such codes for several years in the past. It was finally established that there are no perfect codes other than the single error correcting Hamming [5] codes, double and triple error correcting Golay codes [7], and the Repetitive codes (refer to Tietavainen [8], Tietavainen and Perko [9], and van Lint [10]).

By perfect codes we mean the linear codes that are capable of correcting all $t$ or fewer errors and no others.

Thereafter several attempts were given to find codes that are not perfect in the usual sense but that correct certain type of error pattern and no more. Such codes are called optimal codes. Sharma and Dass [11] were the first who attempted to find such codes. In paper [12], Dass and Tyagi explored a new type of binary $(1,2)$ optimal codes. Similar kind of perfect codes is also studied in [13].

Further, mathematicians also started to find codes that are opposite in nature to perfect codes. Those codes are called anti-perfect codes. In this direction, an attempt is given in paper [14] by Sharma et al. These codes correct all $t$ errors and more and no others.

In view of these studies, this paper presents $\left(n=n_{1}+\right.$ $n_{2}, k$ ) linear optimal codes over $\mathrm{GF}(q)$ that correct all periodic errors of order $r$ in the first block of length $n_{1}$ and all periodic errors of order $s$ in the second block of length $n_{2}$ and no 
others. Then the study has been extended to the case when the errors in the first block of length $n_{1}$ are in the form of order $r$ or more and the errors in the second block of length $n_{2}$ are in the form of order $s$ or more and no others. They are called anti-optimal codes.

This paper also presents the weight structure of periodic errors in the space of $n$-tuples over $\operatorname{GF}(q)$. The study of weight structure for different types of error patterns is of considerable interest to many researchers. Various results are obtained in this direction (e.g., $[15,16])$.

The paper is organized as follows. Section 1 is the introduction. In Section 2, we present minimum weight and weight structure of periodic errors in the space of $n$-tuples. In Section 3, we study optimal codes that correct all periodic errors of order $r$ in the first block of length $n_{1}$ and all periodic errors of order $s$ in the second block of length $n_{2}$ and no others. Section 4 presents the study of anti-optimal codes mentioned above.

\section{Weights of Periodic Errors}

In coding theory, an important criterion is to look for minimum weight and structure of weight in a group of vectors. Our following theorems (which are equivalent to Plotkin bound [17], also Theorem 4.1, Peterson and Weldon [6]) are results in that direction. The weight of a vector is considered in Hamming's sense.

Lemma 2. Let $W_{s}$ denote the total weight of all periodic errors of order $s$ in the space of all $n$-tuples over $G F(q)$. Then,

$$
\begin{aligned}
W_{s}= & \sum_{w=1}^{k_{0}} w\left(\begin{array}{l}
k_{0} \\
w
\end{array}\right)(q-1)^{w}+\sum_{w=1}^{k_{1}} w\left(\begin{array}{l}
k_{1} \\
w
\end{array}\right)(q-1)^{w} \\
& +\cdots+\sum_{w=1}^{k_{s}} w\left(\begin{array}{c}
k_{s} \\
w
\end{array}\right)(q-1)^{w},
\end{aligned}
$$

where $k_{i}=\lceil(n-i) /(s+1)\rceil(i=0,1,2, \ldots s)$.

Proof. We first count the total number of periodic errors of order $s$ with weight $w$ in the space of all $n$-tuples.

Consider a periodic error of order $s$. The number of positions in which periodic error of order $s$ can occur is $k_{0}, k_{1}, \ldots, k_{s}$ where $k_{i}=\lceil(n-i) /(s+1)\rceil(i=0,1,2, \ldots, s)$ and $w \leq k_{s}$ (refer to Tyagi and Das [4]). So, the total number of periodic errors of order $s$ with weight $w$ is given by

$$
\left\{\left(\begin{array}{c}
k_{0} \\
w
\end{array}\right)+\left(\begin{array}{c}
k_{1} \\
w
\end{array}\right)+\cdots+\left(\begin{array}{c}
k_{s} \\
w
\end{array}\right)\right\}(q-1)^{w} .
$$

Then,

$$
\begin{aligned}
W_{s}= & \sum_{w=1}^{k_{0}} w\left(\begin{array}{l}
k_{0} \\
w
\end{array}\right)(q-1)^{w}+\sum_{w=1}^{k_{1}} w\left(\begin{array}{l}
k_{1} \\
w
\end{array}\right)(q-1)^{w} \\
& +\cdots+\sum_{w=1}^{k_{s}} w\left(\begin{array}{l}
k_{s} \\
w
\end{array}\right)(q-1)^{w} .
\end{aligned}
$$

Theorem 3. The minimum weight of a periodic error of order $s$ in the space of $n$-tuples is at most

$$
\begin{gathered}
\left(\sum_{w=1}^{k_{0}} w\left(\begin{array}{c}
k_{0} \\
w
\end{array}\right)(q-1)^{w}+\sum_{w=1}^{k_{1}} w\left(\begin{array}{l}
k_{1} \\
w
\end{array}\right)(q-1)^{w}\right. \\
\left.+\cdots+\sum_{w=1}^{k_{s}} w\left(\begin{array}{c}
k_{s} \\
w
\end{array}\right)(q-1)^{w}\right) \\
\times\left(\sum_{i=0}^{s}\left(q^{k_{i}}-1\right)\right)^{-1}
\end{gathered}
$$

where $k_{i}=\lceil(n-i) /(s+1)\rceil, i=0,1,2, \ldots s$.

Proof. The number of periodic errors of order $s$ in the space of $n$-tuples over $\mathrm{GF}(q)$ is given by

$$
\sum_{i=0}^{s}\left(q^{k_{i}}-1\right), \quad \text { where } k_{i}=\left\lceil\frac{n-i}{s+1}\right\rceil(i=0,1,2, \ldots, s) .
$$

By using Lemma 2, the total weight of all periodic errors of order $s$ is given by

$$
\begin{gathered}
\sum_{w=1}^{k_{0}} w\left(\begin{array}{c}
k_{0} \\
w
\end{array}\right)(q-1)^{w}+\sum_{w=1}^{k_{1}} w\left(\begin{array}{l}
k_{1} \\
w
\end{array}\right)(q-1)^{w} \\
+\cdots+\sum_{w=1}^{k_{s}} w\left(\begin{array}{c}
k_{s} \\
w
\end{array}\right)(q-1)^{w} .
\end{gathered}
$$

Since the minimum weight element can have at most the average weight, an upper bound on the minimum weight of periodic errors of order $s$ is given by

$$
\begin{gathered}
\left(\sum_{w=1}^{k_{0}} w\left(\begin{array}{c}
k_{0} \\
w
\end{array}\right)(q-1)^{w}+\sum_{w=1}^{k_{1}} w\left(\begin{array}{l}
k_{1} \\
w
\end{array}\right)(q-1)^{w}\right. \\
\left.+\cdots+\sum_{w=1}^{k_{s}} w\left(\begin{array}{c}
k_{s} \\
w
\end{array}\right)(q-1)^{w}\right) \\
\times\left(\sum_{i=0}^{s}\left(q^{k_{i}}-1\right)\right)^{-1} .
\end{gathered}
$$

During the process of transmission, periodic disturbances cause occurrence of periodic errors. But it is quite possible that all the periodic components in such periodic errors may not be affected; that is, some digits are received correctly while others get corrupted. In view of this, we have the following results for periodic errors with weight $w$ or less (without proof). 
Lemma 4. Let $W_{s, w}$ denote the total weight of all periodic errors of order $s$ which are of weight $w$ or less $\left(w \leq k_{s}\right)$ in the space of all $n$-tuples. Then,

$$
\begin{aligned}
W_{s, w}= & \sum_{m=1}^{w} m\left(\begin{array}{l}
k_{0} \\
m
\end{array}\right)(q-1)^{m}+\sum_{m=1}^{w} m\left(\begin{array}{l}
k_{1} \\
m
\end{array}\right)(q-1)^{m} \\
& +\cdots+\sum_{m=1}^{w} m\left(\begin{array}{l}
k_{s} \\
m
\end{array}\right)(q-1)^{m},
\end{aligned}
$$

where $k_{i}=\lceil(n-i) /(s+1)\rceil, i=0,1,2, \ldots s$.

Theorem 5. The minimum weight of a periodic error of order $s$ which is of weight $w$ or less $\left(w \leq k_{s}\right)$ in the space of $n$-tuples is at most

$$
\begin{gathered}
\left(\sum_{m=1}^{w} m\left(\begin{array}{l}
k_{0} \\
m
\end{array}\right)(q-1)^{m}+\sum_{m=1}^{w} m\left(\begin{array}{l}
k_{1} \\
m
\end{array}\right)(q-1)^{m}\right. \\
\left.+\cdots+\sum_{m=1}^{w} m\left(\begin{array}{c}
k_{s} \\
m
\end{array}\right)(q-1)^{m}\right) \\
\quad \times\left(\sum_{i=0}^{s}\left(q^{k_{i}}-1\right)\right)^{-1},
\end{gathered}
$$

where $k_{i}=\lceil(n-i) /(s+1)\rceil, i=0,1,2, \ldots s$.

\section{Optimal Codes}

Das [18] has studied the $\left(n=n_{1}+n_{2}, k\right)$ linear code over GF( $\left.q\right)$ that corrects all periodic errors of order $r$ in the first block of length $n_{1}$ and all periodic errors of order $s$ in the second block of length $n_{2}$ as follows.

Theorem 6. The number of parity check digits for an $\left(n=n_{1}+\right.$ $\left.n_{2}, k\right)$ linear code over $G F(q)$ that corrects all periodic errors of order $r$ in the first block of length $n_{1}$ and all periodic errors of order $s$ in the second block of length $n_{2}$ always satisfies

$$
q^{n-k} \geq 1+\sum_{i=0}^{r}\left(q^{k_{i}^{r}}-1\right)+\sum_{j=0}^{s}\left(q^{k_{j}^{s}}-1\right)
$$

where $k_{i}^{r}=\left\lceil\left(n_{1}-i\right) /(r+1)\right\rceil(i=0,1,2, \ldots r)$ and $k_{j}^{s}=\left\lceil\left(n_{2}-\right.\right.$ $j) /(s+1)\rceil(j=0,1,2, \ldots s)$.

Considering the equality of inequality (10) gives us the optimal case; that is,

$$
q^{n-k}=1+\sum_{i=0}^{r}\left(q^{k_{i}^{r}}-1\right)+\sum_{j=0}^{s}\left(q^{k_{j}^{s}}-1\right),
$$

where $k_{i}^{r}=\left\lceil\left(n_{1}-i\right) /(r+1)\right\rceil, k_{j}^{s}=\left\lceil\left(n_{2}-j\right) /(s+1)\right\rceil,(i=$ $0,1,2, \ldots r),(j=0,1,2, \ldots s)$.

We now give an example of a linear code over $\mathrm{GF}(2)$ that corrects all periodic errors of order 2 in the first block of length 6 and all periodic errors of order 1 in the second block of length 4 and no other errors.
TABLE 1: Error pattern syndromes.

\begin{tabular}{lccc}
\hline Error patterns & Syndromes & Error patterns & Syndromes \\
\hline 1001000000 & 1100 & 0000010000 & 0101 \\
0100100000 & 1001 & 0000001010 & 0011 \\
0010010000 & 1111 & 0000000101 & 1011 \\
1000000000 & 1000 & 0000001000 & 0010 \\
0100000000 & 1110 & 0000000100 & 0110 \\
0010000000 & 1010 & 0000000010 & 0001 \\
0001000000 & 0100 & 0000000001 & 1101 \\
0000100000 & 0111 & & \\
\hline
\end{tabular}

Example 7. By putting $r=2, s=1, n_{1}=6$, and $n_{2}=4$ over $\mathrm{GF}(2)$, equality $(11)$ gives rise to $(6+4,6)$ linear code. Consider the following matrix:

$$
H=\left[\begin{array}{ll}
111000 & 0001 \\
010111 & 0101 \\
011010 & 1100 \\
000011 & 0011
\end{array}\right]
$$

The code obtained from the above matrix $H$ as a parity check matrix is a $(6+4,6)$ linear code. This code can correct all periodic errors of order 2 in the first block of length 6 and all periodic errors of order 1 in the second block of length 4 and no others. We list in Table 1 all the error vectors and their corresponding syndromes which can be seen to be all distinct and exhaustive.

\section{Anti-Optimal Codes}

In this section, we will obtain bound on $\left(n=n_{1}+n_{2}, k\right)$ linear code over $\operatorname{GF}(q)$ that corrects all periodic errors of order $r$ or more in the first block of length $n_{1}\left(r \geq\left(n_{1}-3\right) / 2\right)$ and all periodic errors of order $s$ or more in the second block of length $n_{2}\left(s \geq\left(n_{2}-3\right) / 2\right)$ and no other errors. Taking the bound tight, we obtain anti-optimal codes. The codes are anti-optimal codes in the sense that they correct all periodic errors of order $r$ or more in the first block of length $n_{1}$ and all periodic errors of order $s$ or more in the second block of length $n_{2}$ and no others. First we prove the following lemma.

Lemma 8. If $B(n, s)$ (where $n \leq 2 s+3$ ) denotes the number of periodic errors of order $s$ or more over the space of all $n$-tuples over $G F(q)$, then

$$
B(n, s)= \begin{cases}\sum_{i=s}^{n-1} E(i)-(n-s-1) n(q-1), & \text { for } n \leq 2 s+1, \\ \sum_{i=s}^{2 s} E(i)-\operatorname{sn}(q-1), & \text { for } n=2 s+3\end{cases}
$$

where $E(i)=\sum_{j=0}^{i}\left(q^{k_{j}^{i}}-1\right)$ and $k_{j}^{i}=\lceil(n-j) /(i+1)\rceil$. 
Proof. For $n \leq 2 s+1$, there will be no common errors among the periodic errors of order $s$ or more except the single errors. Let $E(s)$ be the number of periodic errors of order $s$. Then,

$$
E(s)=\sum_{j=0}^{s}\left(q^{k_{j}^{s}}-1\right), \quad \text { where } k_{j}^{s}=\left\lceil\frac{n-j}{s+1}\right\rceil
$$

(refer to Tyagi and Das [4]).

Therefore

$$
\begin{aligned}
B(n, s)= & E(s)+E(s+1)+\cdots+E(n-2) \\
& +E(n-1)-(n-s-1) n(q-1) \\
= & \sum_{i=s}^{n-1} E(i)-(n-s-1) n(q-1),
\end{aligned}
$$

where

$$
E(i)=\sum_{j=0}^{i}\left(q^{k_{j}^{i}}-1\right), \quad k_{j}^{i}=\left\lceil\frac{n-j}{i+1}\right\rceil .
$$

Let $n=2 s+2$. Since any periodic error of order $2 s+1$ is a periodic error of order $s$, therefore we have

$$
\begin{aligned}
B(n, s)= & E(s)+E(s+1)+\cdots \\
& +E(2 s)-s n(q-1) \\
= & \sum_{i=s}^{2 s} E(i)-s n(q-1) .
\end{aligned}
$$

Let $n=2 s+3$. Since $E(n-1)$ represents the single errors and all single errors present in periodic errors of any order, so by counting the number of periodic errors of order $s$ or more, we take the value of $s$ up to $n-2$. Also, any periodic error of order $2 s+1$ is a periodic error of order $s$. Hence

$$
\begin{aligned}
B(n, s) & =E(s)+E(s+1)+\cdots+E(2 s)-s n(q-1) \\
& =\sum_{i=s}^{2 s} E(i)-s n(q-1) .
\end{aligned}
$$

Theorem 9. The number of parity check digits for an $\left(n=n_{1}+\right.$ $\left.n_{2}, k\right)$ linear code over $G F(q)$ that corrects all periodic errors of order $r$ or more in the first block of length $n_{1}\left(r \geq\left(n_{1}-3\right) / 2\right)$ and all periodic errors of order $s$ or more in the second block of length $n_{2}\left(s \geq\left(n_{2}-3\right) / 2\right)$ and no other error patterns is at least

$$
\log _{q}\left[1+B\left(n_{1}, r\right)+B\left(n_{2}, s\right)\right]
$$

where $B\left(n_{1}, r\right)$ and $B\left(n_{2}, s\right)$ are given in Lemma 8 .

Proof. This proof is based on counting the number of errors above specific type and comparing with the available cosets in the $\left(n=n_{1}+n_{2}, k\right)$ linear code over $\mathrm{GF}(q)$.
TABLE 2: Error pattern syndromes.

\begin{tabular}{lccc}
\hline Error patterns & Syndromes & Error patterns & Syndromes \\
\hline 100000000 & 1110 & 000001001 & 1111 \\
010000000 & 1011 & 000100010 & 1001 \\
001000000 & 1110 & 000010001 & 0111 \\
000100000 & 1000 & 000100001 & 1101 \\
000100100 & 1100 & 000000100 & 0100 \\
000010000 & 0010 & 000000010 & 0001 \\
000010010 & 0011 & 000000001 & 0101 \\
000001000 & 1010 & & \\
\hline
\end{tabular}

By Lemma 8, we have the following.

(a) The number of periodic errors of order $r$ or more in the first block of length $n_{1}$ is $B\left(n_{1}, r\right)$.

(b) The number of periodic errors of order $s$ or more in the second block of length $n_{2}$ is $B\left(n_{2}, s\right)$.

Therefore, the total number of errors including the zero vector is

$$
1+B\left(n_{1}, r\right)+B\left(n_{2}, s\right)
$$

Thus

$$
q^{n-k} \geq 1+B\left(n_{1}, r\right)+B\left(n_{2}, s\right) .
$$

Hence the proof of Theorem 9 is complete.

Now the equality of inequality (21) gives us the optimal case. By considering the equality in (21), we get

$$
q^{n-k}=1+B\left(n_{1}, r\right)+B\left(n_{2}, s\right) .
$$

For $r=n_{1}-1$ and $q=2$, (22) becomes

$$
2^{n-k}=1+n_{1}+B\left(n_{2}, s\right) \text {. }
$$

Example 10. For $n_{1}=3, n_{2}=6$, and $s=2$, (23) gives rise to binary $(3+6,5)$ linear code. The code whose parity check matrix is given below is a periodic error correcting antioptimal code that corrects all periodic errors of order 2 or more in the first block of length 3 and all periodic errors of order 2 or more in the second block of length 6 and no others. Consider

$$
H=\left[\begin{array}{ll}
111 & 101000 \\
101 & 000101 \\
111 & 011000 \\
010 & 000011
\end{array}\right]
$$

It can be verified from the error pattern syndromes shown in Table 2.

Example 11. For $n_{1}=6, n_{2}=9$, and $s=3$, (23) gives rise to binary $(6+9,10)$ linear code. The code whose parity check matrix is given below is a periodic error correcting antioptimal codes in two blocks that correct all single errors in 
the first block of length 6 and all periodic errors of order 3 or more in the second block of length 9 and no more. It can also be verified by the error pattern syndrome table. Consider

$$
H=\left[\begin{array}{ll}
011000 & 101100000 \\
000110 & 001110110 \\
010100 & 000101011 \\
111110 & 010100110 \\
101001 & 001001110
\end{array}\right] .
$$

\section{Conflict of Interests}

The author declares that there is no conflict of interests regarding the publication of this paper.

\section{Acknowledgment}

The author would like to thank referees for their careful reading of the paper and for their valuable suggestions.

\section{References}

[1] "Astrophotography mounts: periodic error correction," http://www.themcdonalds.net/richard/index.php?title=Main Page.

[2] V. Tyagi and A. Tyagi, "Periodic error correcting perfect codes," International Journal of Computer and Information Technology, vol. 2, no. 5, pp. 603-607, 2013.

[3] P. K. Das and V. Tyagi, "Codes on s-periodic errors," Ratio Mathematica-Journal of Applied Mathematics, vol. 22, pp. 6168, 2012.

[4] V. K. Tyagi and P. K. Das, "s-alternate error correcting linear code," Journal of Combinatorics, Information \& System Sciences, vol. 35, no. 1-2, pp. 17-26, 2010.

[5] R. W. Hamming, "Error detecting and error correcting codes," The Bell System Technical Journal, vol. 29, pp. 147-160, 1950.

[6] W. W. Peterson and J. Weldon, Error-Correcting Codes, MIT Press, Cambridge, Mass, UK, 2nd edition, 1972.

[7] M. J. E. Golay, "Notes on digital coding," Proceedings of the IRE, vol. 37, p. 657, 1949.

[8] A. Tietavainen, "On the non-existence of perfect codes over finite fields," SIAM Journal on Applied Mathematics, vol. 24, no. 1, pp. 88-96, 1971.

[9] A. Tietavainen and A. Perko, "There are no unknown perfect binary codes," Annales Universitatis Turkuensis A, vol. 148, pp. 3-10, 1971.

[10] J. H. van Lint, "A survey of perfect codes," The Rocky Mountain Journal of Mathematics, vol. 5, pp. 199-224, 1975.

[11] B. D. Sharma and B. K. Dass, "Adjacent-error correcting binary perfect codes," Journal of Cybernetics, vol. 7, no. 1-2, pp. 9-13, 1977.

[12] B. K. Dass and V. K. Tyagi, "A new type of $(1,2)$-optimal codes over GF(2)," Indian Journal of Pure and Applied Mathematics, vol. 13, no. 7, pp. 750-756, 1982.

[13] P. K. Das, "(1, 3) Optimal linear codes on solid bursts," International Journal of Information Security Science, vol. 1, no. 4, pp. 106-111, 2012.

[14] B. D. Sharma, B. K. Dass, and S. N. Gupta, "A sufficient condition for the existence of high-density burst correcting linear codes,"
Indian Journal of Pure and Applied Mathematics, vol. 8, no. 7, pp. 816-820, 1977.

[15] B. D. Sharma and B. K. Dass, "On weight of bursts," in Proceedings of the 38th Annual Conference of IMS, Bhopal, India, 1972.

[16] B. D. Sharma and B. Rohtagi, "Some results on weights of vectors having $m$-repeated bursts," Cybernetics and Information Technologies, vol. 11, no. 3, pp. 3-11, 2011.

[17] M. Plotkin, "Binary code with specified minimum weight," IRE Transactions on Information Theory, vol. 6, pp. 440-450, 1960.

[18] P. K. Das, "Bounds on codes correcting periodic errors blockwise," International Journal of Informatics and Communication Technology, vol. 2, no. 1, pp. 51-56, 2013. 


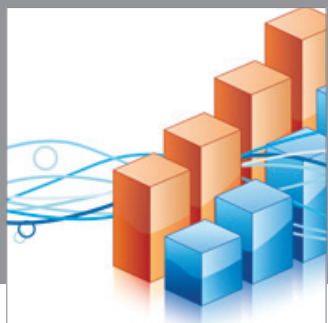

Advances in

Operations Research

mansans

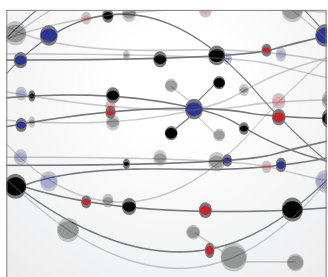

The Scientific World Journal
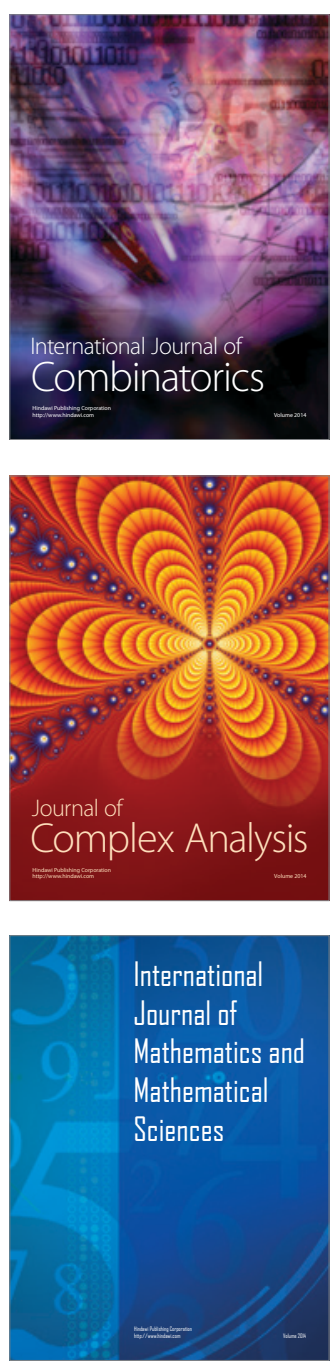
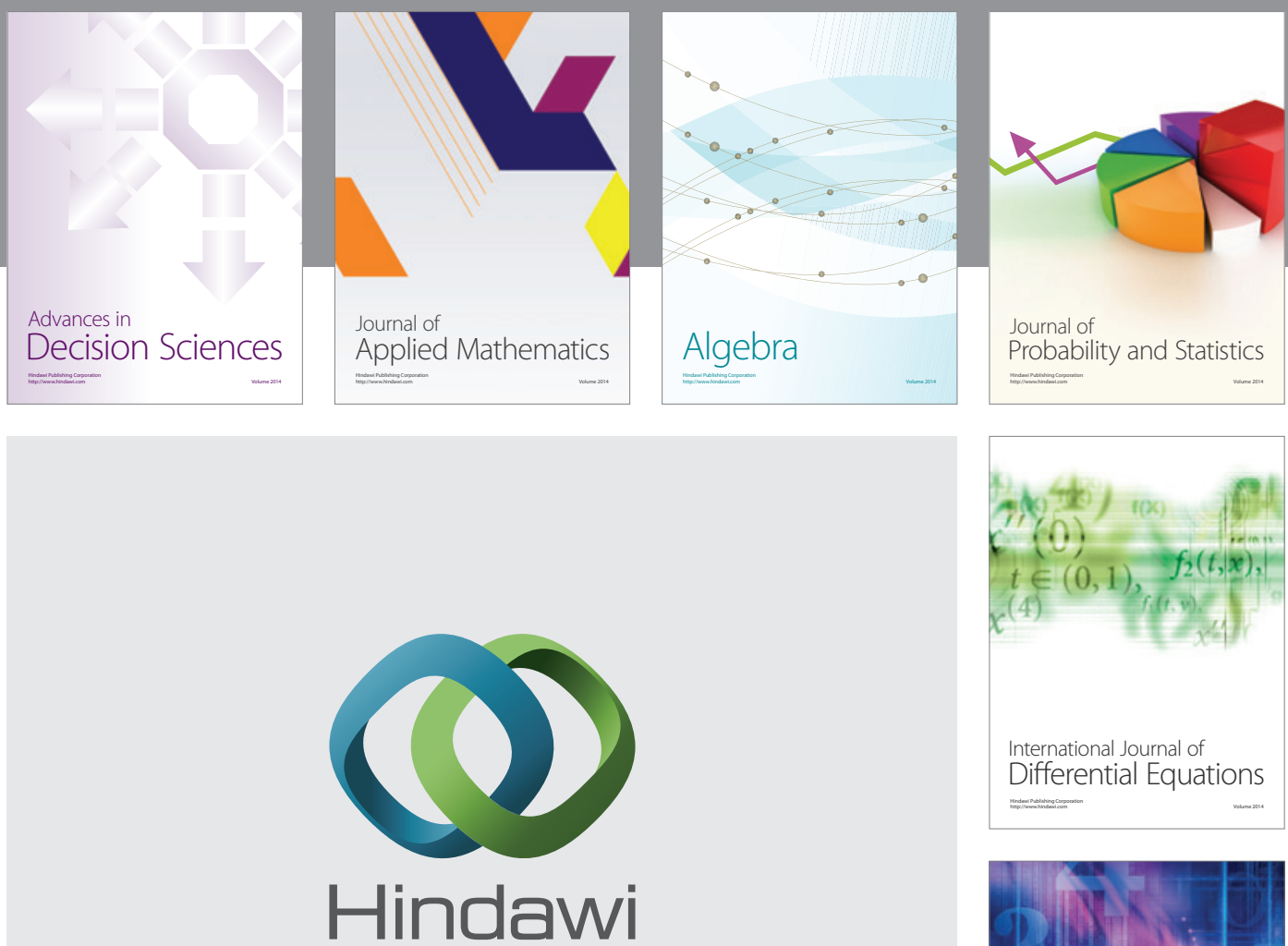

Submit your manuscripts at http://www.hindawi.com
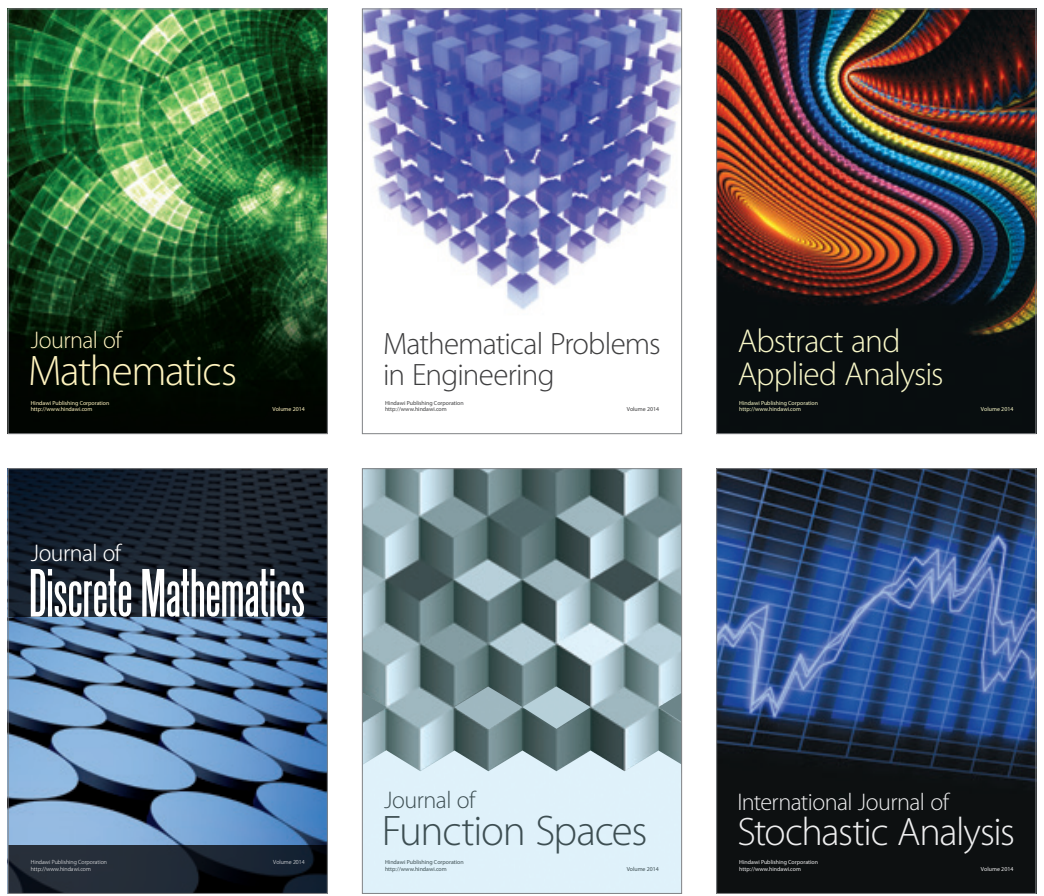

Journal of

Function Spaces

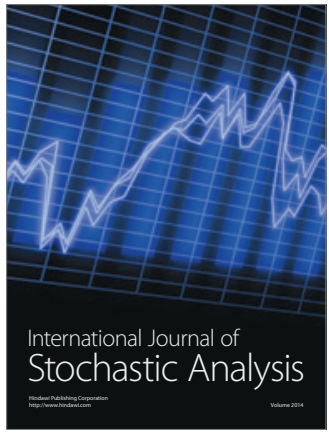

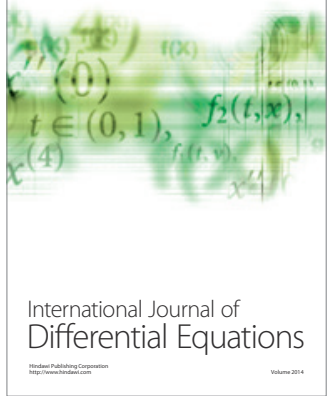
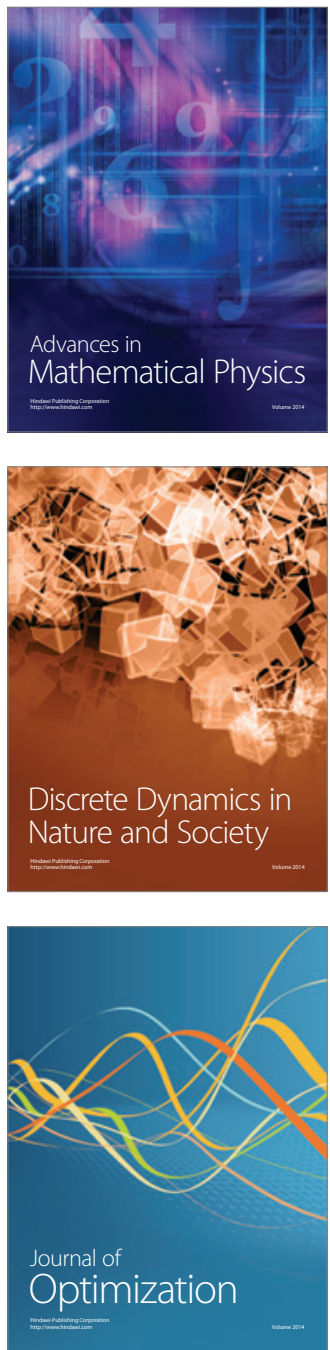